\title{
Scattering into Cones I: Potential Scattering
}

\author{
John Day Dollard* \\ Department of Mathematics, University of Rochester, Rochester, New York \\ Received August 16, 1968
}

\begin{abstract}
In the non-relativistic time-dependent theory of scattering, we compute the probability that a particle with given initial state should scatter into a cone with apex at the origin. A formula for this probability is found which holds good for a large class of short-range potentials and also for Coulomb potentials. The formula is obtained by showing that if the initial state of a particle is specified by $f$, then its position probability density at large positive times can be taken to be $\left|\left(e^{-2 i H t} e^{i H_{o} t} f\right)(\boldsymbol{x})\right|^{2}$.
\end{abstract}

\section{Introduction}

In the time-independent theory of non-relativistic potential scattering, the principal object of interest is the differential scattering crosssection $\sigma(\theta, \emptyset)$. This quantity has a very simple interpretation, as it is the probability density that an incident particle will be deflected through a certain angle during the scattering process. Typically, in the timedependent theory, one loses sight of the simple geometrical features of the scattering process and concentrates attention on the computation of transition probabilities from one state to another. In this paper, we will emphasize geometrical features in the time-dependent theory by computing the probability that a particle with given initial wave-function will be scattered into a cone $C$ with apex at the origin of coordinates. This quantity has an easily visualizable meaning, and it seems a natural thing to calculate in view of typical experimental setups. The formula obtained for this probability can be put in a simple form which is applicable both to scattering problems involving a large class of short-range potentials and to scattering by Coulomb potentials. The formula also admits of a generalization to problems involving $n$ particles. In this paper we discuss the case of potential scattering. $n$-body problems will be dealt with in a forthcoming paper.

\section{Potential Scattering into Cones}

Orientation. We describe a nonrelativistic particle by a wave-function, i.e. a normalized element $\Psi$ of $\mathscr{L}^{2}\left(\mathbb{R}^{3}\right)$, the Hilbert space of complex valued square-integrable functions on three-dimensional Euclidean

* The author wishes to acknowledge the support during this work of NSF Grant GP 7453. 
space. $|\Psi(\boldsymbol{x})|^{2}$ is the position probability density (ppd) for the particle, and if $\widetilde{\Psi}$ is the Fourier transform of $\Psi$, then $|\widetilde{\Psi}(\boldsymbol{k})|^{2}$ is the momentum probability density (mpd) for the particle. If the particle has mass $m$, then the Hamiltonian governing the motion of the particle has the form $(\hbar=1)$

$$
H=\frac{-\Delta}{2 m}+V=H_{o}+V,
$$

where $\Delta$ is the (natural self-adjoint extension of the) Laplacean operator and $V$ is the operator consisting of multiplication by the real function $V(\boldsymbol{x})$. Under appropriate conditions on $V$ (it suffices that $V$ can be written as the sum of a bounded function and a square-integrable function), $H$ is self-adjoint with the same domain as $H_{0}$ [1]. The wavefunction $\Psi_{t}$ of the particle develops in time according to

$$
\Psi_{t}=e^{-i H t} \Psi_{o},
$$

and we shall be interested in the asymptotic behavior of the ppd determined by $\Psi_{t}$ when $t \rightarrow \pm \infty$.

We begin with some simple remarks about the behavior of a free particle. In particular we shall factor the free time-propagation operator $e^{-i H_{o} t}$ into two parts in order to study easily the asymptotic behavior of the ppd of a free particle.

Lemma 1. For $t \neq 0$, define the operators $C_{t}$ and $Q_{t}$ by

$$
\left(C_{t} f\right)(\boldsymbol{x})=\left(\frac{m}{i t}\right)^{3 / 2} e^{\mathrm{im} x^{2} / 2 t} \tilde{f}\left(\frac{m \boldsymbol{x}}{t}\right)
$$

and

$$
\left(Q_{t} f\right)(\boldsymbol{x})=e^{\mathrm{im} x^{2} / 2 t} f(\boldsymbol{x}) .
$$

Then $C_{t}$ and $Q_{t}$ are unitary, and for $t \neq 0$ we have

$$
e^{-i H_{0} t}=C_{t} Q_{t} \text {. }
$$

Proof. It is easy to check that $C_{t}$ and $Q_{t}$ are unitary. Hence all operators occurring in (5) are continuous, and we need only verify that (5) holds on a set dense in $\mathscr{L}^{2}$, which we take to be Schwartz' space [2] $\mathscr{S}$ of $C^{\infty}$ functions which, along with all their derivatives, vanish rapidly at large values of $|\boldsymbol{x}|$. But if $f \in \mathscr{S}$, one need only write down the integral defining the Fourier transform in $C_{t}$ and rearrange to find

$$
\left(C_{t} Q_{t} f\right)(\boldsymbol{x})=\left(\frac{m}{2 \pi i t}\right)^{3 / 2} \int e^{\mathrm{im}\left(\boldsymbol{x}-\boldsymbol{x}^{\prime}\right)^{2} / 2 t} f(\boldsymbol{x}) d \boldsymbol{x} .
$$

The right-hand side of $(6)$ is the well-known integral representation for $\left(e^{-i H_{o} t} f\right)(\boldsymbol{x})$, and this completes the proof of the lemma.

Lemma 2. Let $f \in \mathscr{L}^{2}$. Then

$$
\lim _{t \rightarrow \pm \infty}\left\|e^{-i H_{o} t} f-C_{t} f\right\|=0 .
$$


Proof. By Lemma 1 , and using the unitarity of $C_{t}$, we have for $t \neq 0$,

$$
\begin{aligned}
\left\|e^{-i H_{0} t} f-C_{t} f\right\|^{2} & =\left\|Q_{t} f-f\right\|^{2} \\
& =\int\left|e^{\mathrm{im} x^{2} / 2 t}-1\right|^{2}|f(\boldsymbol{x})|^{2} d \boldsymbol{x},
\end{aligned}
$$

and the last integral approaches zero as $t \rightarrow \pm \infty$ by LEBESGUE's dominated convergence theorem, proving the lemma.

Lemma 3. Let $g_{t}$ and $h_{t}$ belong to $\mathscr{L}^{2}$ and let $\left\|g_{t}\right\|=\left\|h_{t}\right\|=1$. Further suppose that $\lim _{t \rightarrow \pm \infty}\left\|g_{t}-h_{t}\right\|=0$. Let $S$ be a measurable subset of $\mathbb{R}^{3}$. Then

$$
\lim _{t \rightarrow \pm \infty} \int_{S}\left|g_{t}(\boldsymbol{x})\right|^{2} d \boldsymbol{x}=\lim _{t \rightarrow \pm \infty} \int_{S}\left|h_{t}(\boldsymbol{x})\right|^{2} d \boldsymbol{x},
$$

in the sense that the limit on the left-hand side exists if and only if the limit on the right-hand side exists, and when both exist they are equal.

Proof. As is easily seen, it suffices to show that the quantity $\left.\int_{S}|| g_{t}(\boldsymbol{x})\right|^{2}-\left|h_{t}(\boldsymbol{x})\right|^{2} \mid d \boldsymbol{x}$ approaches zero as $t \rightarrow \pm \infty$. But

$$
\begin{aligned}
&\left.\int_{S}|| g_{t}(\boldsymbol{x})\right|^{2}-\left|h_{t}(\boldsymbol{x})\right|^{2} \mid d \boldsymbol{x} \\
& \leqq\left.\int_{\mathbb{R}^{3}}|| g_{t}(\boldsymbol{x})\right|^{2}-\left|h_{t}(\boldsymbol{x})\right|^{2} \mid d \boldsymbol{x} \\
&=\int_{\mathbb{R}^{3}}\left(\left|g_{t}(\boldsymbol{x})\right|+\left|h_{t}(\boldsymbol{x})\right|\right)|| g_{t}(\boldsymbol{x})|-| h_{t}(\boldsymbol{x}) \| d \boldsymbol{x} \\
& \leqq \int_{\mathbb{R}^{3}}\left(\left|g_{t}(\boldsymbol{x})\right|+\left|h_{t}(\boldsymbol{x})\right|\right)\left|g_{t}(\boldsymbol{x})-h_{t}(\boldsymbol{x})\right| d \boldsymbol{x} \\
& \leqq\left\|\left|g_{t}\right|+\left|h_{t}\right|\right\|\left\|g_{t}-h_{t}\right\| \leqq\left(\left\|g_{t}\right\|+\left\|h_{t}\right\|\right)\left\|g_{t}-h_{t}\right\| \\
&=2\left\|g_{t}-h_{t}\right\| \underset{t \rightarrow \pm \infty}{\longrightarrow} 0,
\end{aligned}
$$

proving the lemma. We have used in (10) some familiar inequalities involving absolute values and the Schwartz and triangle inequalities for functions in $\mathscr{L}^{2}$. We shall express Eq. (9) saying that the ppd determined by $g_{t}$ can asymptotically be replaced by the ppd determined by $h_{t}$.

We can use the above results to obtain an intuitive picture of the asymptotic behavior of a free quantum-mechanical particle as follows: with a free quantum-mechanical particle of mass $m$ described by the wave-function $e^{-i H_{o} t} f, f \in \mathscr{L}^{2}$, we associate a corresponding classical particle, defined to be a free classical particle of mass $m$ which starts from the origin of coordinates at time $t=0$ and has a mpd given by $|\tilde{f}(\boldsymbol{k})|^{2}$, i.e. the same mpd as the quantum mechanical particle under consideration. The ppd at time $t$ for the corresponding classical particle is easy to calculate, and is found to be $\left|\frac{m}{t}\right|^{3}\left|\tilde{f}\left(\frac{m \boldsymbol{x}}{t}\right)\right|^{2}(t \neq 0)$. A glance at Eq. (3) shows that this is the same as $\left|\left(C_{t} f\right)(\boldsymbol{x})\right|^{2}$. Taking $g_{t}$ and $h_{t}$ as $e^{-i H_{0} t} f$ and $C_{t} f$ respectively in Lemma 3 , we then find (because of Lemma 2)

$$
\lim _{t \rightarrow \pm \infty} \int_{S}\left|\left(e^{-i H_{0} t} f\right)(\boldsymbol{x})\right|^{2} d \boldsymbol{x}=\lim _{t \rightarrow \pm \infty}\left|\frac{m}{t}\right|^{3} \int_{S}\left|\tilde{f}\left(\frac{m \boldsymbol{x}}{t}\right)\right|^{2} d \boldsymbol{x},
$$


and this equation says that in any calculation of the position of the quantum-mechanical particle, the ppd may asymptotically be replaced by the ppd for the corresponding classical particle. This fact makes it easy to picture the asymptotic motion of the quantum-mechanical particle. We shall in the sequel refer to $C_{t}$ of Eq. (3) as the classical transformation, because $\left|\left(C_{t} f\right)(\boldsymbol{x})\right|^{2}$ is the ppd for the corresponding classical particle.

We now introduce a cone $C$ with apex at the origin of coordinates. $C$ is defined as the set of all points $\boldsymbol{x} \in \mathbb{R}$ satisfying

$$
\boldsymbol{x} \cdot \boldsymbol{n} \geqq \alpha|\boldsymbol{x}|
$$

where $\boldsymbol{n}$ is a unit vector and $0<\alpha \leqq 1$. Along with $C$, we define the cone $-C$ which is the reflection of $C$ through the origin, i.e.

$$
-C=\{-\boldsymbol{x} \mid \boldsymbol{x} \in C\} .
$$

(In the sequel, we shall occasionally denote $C$ by $+C$.) We shall compute the asymptotic probability that a free quantum-mechanical particle will be found in the cone $C$ :

Lemma 4. If a quantum-mechanical particle is described by the wavefunction $e^{-i H_{o} t} f, f \in \mathscr{L}^{2}$, then the asymptotic probability for finding this particle in the cone $C$ as $t \rightarrow \pm \infty$ is given by

$$
\lim _{t \rightarrow \pm \infty} \int_{C}\left|\left(e^{-i H_{0} t} f\right)(\boldsymbol{x})\right|^{2} d \boldsymbol{x}=\int_{ \pm C}|\tilde{f}(\boldsymbol{k})|^{2} d \boldsymbol{k} .
$$

Proof. The required probability is the left-hand side of (14). By (11), the integrand on the left-hand side of (14) can be replaced by $\left|\frac{m}{t}\right|^{3}\left|f\left(\frac{m \boldsymbol{x}}{t}\right)\right|^{2}$. But then the change of variable $\boldsymbol{k}=\frac{m \boldsymbol{x}}{t}$, which carries $C$ into $\pm C$ according as $t>0$ or $t<0$, yields the result immediately.

Lemma 4 is, of course, hardly surprising. It says that the probability for eventually finding the particle in $C$ at large positive times is the same as the probability that its momentum is in $C$, and makes a similar statement about large negative times.

We have now obtained all the formulas we need for our discussion of scattering theory.

To refresh the reader's memory and to set the stage for the discussion to follow, we summarize some facts about the time-dependent theory of scattering. To describe the scattering of a particle whose Hamiltonian is given by Eq. (1), we specify a state of the particle by requiring that at large negative times the wave-function should have the form $e^{-i H_{o} t} f$, with $f \in \mathscr{L}^{2}$. This, of course, is just a manner of speaking, because actually we believe that at all times the wave-function has the form given in Eq. (2), the time-dependence thus always being goverened by the full Hamiltonian. What the requirement really means is this: we 
specify $f \in \mathscr{L}^{2}$ and require that $\Psi_{o}$ of Eq. (2) should be so chosen that

$$
\lim _{t \rightarrow-\infty}\left\|e^{-i H t} \Psi_{o}-e^{-i \Pi_{o} t} f\right\|=0
$$

The function $f$ will be called the initial state of the particle. If for every $f \in \mathscr{L}^{2}$ we are to be able to find a $\Psi_{o}$ satisfying (15), we must require the existence of the Møller wave-matrix

$$
\lim _{t \rightarrow-\infty} e^{i H t} e^{-i H_{0} t}=\Omega^{-} .
$$

Then, if $f$ is given, (15) is satisfied with $\Psi_{o}=\Omega^{-} f$, and clearly this is the only choice of $\Psi_{o}$ which will satisfy (15). Thus, to say that $f$ is the initial state of the particle is the same as saying that the wave-function of the particle at all times is $e^{-i H t} \Omega^{-} f$.

By similar arguments, we are led to require the existence of $\Omega^{+}$, the limit of $e^{i H t} e^{-i H_{0} t}$ as $t \rightarrow+\infty$. A wave-function $e^{-i H t} \Psi_{o}$ converges strongly to $e^{-i H_{0} t} g$ as $t \rightarrow+\infty$ if and only if $\Psi_{o}=\Omega^{+} g . \Omega^{ \pm}$are isometries. We denote by $R^{ \pm}$the range of $\Omega^{ \pm}$. It is natural to believe that $R^{+}$and $R^{-}$are identical, since $R^{-}$is the set of states $\Psi_{o}$ at time $t=0$ describing particles which "become free" as $t \rightarrow-\infty$, and $R+$ is the set describing particles which "become free" as $t \rightarrow+\infty$. In the sequel, we shall assume that the potential $V$ occuring in (1) is such that $\Omega^{ \pm}$exist and $R^{+}=R^{-}$. (For this to be true it suffices [3] that $V \in \mathscr{L}^{1}\left(\mathbb{R}^{3}\right)$ $\cap \mathscr{L}^{2}\left(\mathbb{R}^{3}\right)$.) We shall denote $R^{ \pm}$by $R$. Denoting by $P_{R}$ the projection on $R$, we have

$$
P_{R}=\Omega^{ \pm} \Omega^{ \pm *}
$$

If the wave-function of a particle is specified by the initial state $f$, then the wave-function $\Psi_{o}$ at $t=0$ is equal to $\Omega^{-} f$, which belongs to $R$, and writing

$$
\Psi_{o}=\Omega^{-} f=P_{R} \Omega^{-} f=\Omega^{+} \Omega^{+*} \Omega^{-} f
$$

we see that at large positive times the wave-function $e^{-i H t} \Psi_{o}$ converges strongly to $e^{-i H_{0} t} \Omega^{+*} \Omega^{-} f$, or $e^{-i H_{o} t} S f$, where $S$ is the $S$-matrix:

$$
S=\Omega^{+*} \Omega^{-} \text {. }
$$

$S$ is unitary. Infact, this statement is equivalent to the statement $R^{+}=R^{-}$. We now ask this question: given the initial state $f$ for the particle, what is the probability $P(f, C)$ that at large positive times the particle will be found in the cone $C$ ? (We will call this the probability that the particle scatters into $C$.) The answer is now quite simple. According to the above, the probability is given by

$$
P(f, C)=\lim _{t \rightarrow+\infty} \int_{C}\left|\left(e^{-i H t} \Omega^{-} f\right)(\boldsymbol{x})\right|^{2} d \boldsymbol{x} .
$$

We can establish the existence of the limit in (20) and at the same time evaluate the limit as follows: since $R^{+}=R^{-}$, we know by the above 
discussion that $e^{-i H t} \Omega^{-f}$ converges strongly to $e^{-i H_{0} t} S f$ as $t \rightarrow+\infty$, so that, applying Lemma 3, we can asymptotically replace the ppd in the integrand in (20) by the ppd determined by $e^{-i H_{o} t} S f$. Then by Eq. (14) we immediately have

$$
P(f, C)=\int_{C}|\widetilde{S f}(\boldsymbol{k})|^{2} d \boldsymbol{k} .
$$

This result is, of course, just what was to be expected. It has been derived before [4], under somewhat different hypotheses on $V$ and by a method which is unnatural in the present context. The proof given above generalizes easily to $n$-body problems.

We now return to Eq. (20), which we shall generalize slightly in such a way that the generalized equation applies also to scattering by the Coulomb potential. The idea is very simple - in the ppd $e^{-i H t} \Omega^{-} f$ we merely replace $\Omega^{-}$by the expression $e^{-i H t} e^{i H_{o} t}$ which converges to $\Omega^{-}$ as $t \rightarrow+\infty$ (since $e^{i H t} e^{-i H_{o} t}$ converges to $\Omega^{-}$as $t \rightarrow-\infty$ ). We state the result as

Theorem 1. Let a non-relativistic particle have the initial state f. Let the Hamiltonian $H$ describing this particle be such that the strong limits $\Omega^{ \pm}$ exist and $R^{+}=R^{-}$. Then the ppd determined by the wave-function $e^{-i H t} \Omega^{-f}$ of the particle can for large $t$ be replaced by the ppd determined by $e^{-2 i H t} e^{i H_{0} t} f$, i.e.

$$
\lim _{t \rightarrow+\infty} \int_{S}\left|\left(e^{-i H t} \Omega^{-} f\right)(\boldsymbol{x})\right|^{2} d \boldsymbol{x}=\lim _{t \rightarrow+\infty} \int_{S}\left|\left(e^{-2 i H t} e^{i H_{o} t} f\right)(\boldsymbol{x})\right|^{2} d \boldsymbol{x},
$$

where $S$ is any measurable subset of $\mathbb{R}^{3}$. In particular, the probability $P(f, C)$ that the particle scatters into the cone $C$, already computed in Eqs. (20) and (21), is also given by

$$
P(f, C)=\lim _{t \rightarrow+\infty} \int_{C}\left|\left(e^{-2 i H t} e^{i H_{0} t} f\right)(\boldsymbol{x})\right|^{2} d \boldsymbol{x} .
$$

Proof. We have

$$
\begin{aligned}
& \left\|e^{-2 i H t} e^{i H_{0} t} f-e^{-i H t} \Omega^{-} f\right\| \\
= & \left\|e^{-i H t} e^{i H_{0} t} f-\Omega^{-} f\right\| \underset{t \rightarrow+\infty}{\longrightarrow} 0 .
\end{aligned}
$$

By Lemma 3, this shows that the ppd determined by $e^{-i H t} \Omega^{-} f$ can be replaced by that determined by $e^{-2 i H t} e^{i H_{o} t} f$, and the theorem is proved.

With Eq. (22) we have arrived at a formula for $P(f, C)$ which represents a departure from the usual time-dependent scattering theory, because (22) no longer involves the Møller wave matrix $\Omega^{-}$. To be sure, we have derived (22) on the assumption that the Møller wave-matrices exist and $R^{+}=R^{-}$. However, the Møller wave-matrices do not appear in (22) and it is at least conceivable that (22) can be used as the definition of the probability for scattering into $C$ in a case when the Møller wavematrices do not exist, thus providing a simple generalization of nonrelativistic scattering theory. The reader can easily check that in the 
case of a constant potential $V \neq 0$ (in this case the Møller wave-matrices do not exist because $e^{i H t} e^{-i H_{0} t}=e^{i V t}$ does not converge as $t \rightarrow \pm \infty$ ) formula (22) gives the correct result $-P(f, C)$ is the same as for a free particle. In fact, in this case the ppd determined by $e^{-2 i H t} e^{i H_{0} t} f$ is identical with that determined by the solution $e^{-i H_{0} t} f$ of the free Schrödinger equation. It might be objected, however, that the case of a constant potential has never been a troublesome one, and that the constant should be set equal to zero so that the Møller wave-matrices do exist. We therefore proceed to consider a harder case, and one of great physical interest: we take $V$ to be a Coulomb potential.

For the convenience of the reader we recollect some facts about scattering by a Coulomb potential. If the Hamiltonian for the particle to be scattered is

$$
H=\frac{-\Delta}{2 m}+\frac{e_{1} e_{2}}{|\boldsymbol{x}|},
$$

then it is no longer consistent to assume that at large positive and negative times the wave-function $e^{-i H t} \Psi_{o}$ of the particle converges strongly to functions of the form $e^{-i H_{o} t} f$, with $f \in \mathscr{L}^{2}$. Instead, the wavefunction describing a scattering process converges strongly at large positive and negative times to functions of the form $e^{-i H_{o c}(t)} g$, with $g \in \mathscr{L}^{2}$, where [5]

with

$$
H_{o c}(t)=H_{o} t+H_{o c}{ }^{\prime}(t)
$$

and

$$
H_{o c}^{\prime}(t)=\epsilon(t) \frac{m e_{1} e_{2}}{(-\Delta)^{1 / 2}} \quad \log \left(\frac{-2|t| \Delta}{m}\right)
$$

$$
\epsilon(t)=\left\{\begin{array}{rr}
1 & (t>0) \\
-1 & (t<0) .
\end{array}\right.
$$

Although $e^{-i H_{o c}(t)} g$ is not a solution of the free Schrödinger equation, it is still possible to prove [5] that for any measurable subset $S$ of $\mathbb{R}^{3}$ we have

$$
\begin{aligned}
& \lim _{t \rightarrow \pm \infty} \int_{S}\left|\left(e^{-i H_{o c}(t)} g\right)(\boldsymbol{x})\right|^{2} d \boldsymbol{x} \\
= & \lim _{t \rightarrow \pm \infty}\left|\frac{m}{t}\right|^{3} \int_{S}\left|\tilde{g}\left(\frac{m \boldsymbol{x}}{t}\right)\right|^{2} d \boldsymbol{x},
\end{aligned}
$$

so that asymptotically the ppd determined by $e^{-i H_{o c}(t)} \mathrm{g}$ can be replaced by that for the solution $e^{-i H_{0} t} g$ of the free Schrödinger equation. We can thus think of the particle as travelling freely at large times. If the wave-function $e^{-i H t} \Psi_{o}$ of a particle converges strongly at large negative times to $e^{-i H_{o c}(t)} f$, we shall refer to $f$ as the initial state of the particle.

The strong limits

$$
\lim _{t \rightarrow \pm \infty} e^{i H t} e^{-i H_{o c}(t)}=\Omega_{c}^{ \pm}
$$


exist and are isometric. If $f$ is the initial state of a particle, then its wave-function at all times is $e^{-i H t} \Omega_{c}^{-} f$. The ranges of the operators $\Omega_{c}^{ \pm}$are the same, and just as before it follows that if the initial state of the particle is $f$, then at large positive times the wave-function $e^{-i H t} \Omega_{c}^{-} f$ of the particle converges strongly to $e^{-i H_{o c}(t)} S_{c} f$, where $S_{c}$ is the Coulomb S-matrix:

$$
S_{c}=\Omega_{c}^{+*} \Omega_{c}^{-} .
$$

We can now ask for the probability $P(f, C)$ that a particle with initial state $f$ is scattered into $C$. Using the above discussion, Lemma 3, and (26), we have

just as before.

$$
\begin{aligned}
P(f, C) & \left.=\lim _{t \rightarrow+\infty} \int_{C} \mid e^{-i I t} \Omega_{c}^{-} f\right)\left.(\boldsymbol{x})\right|^{2} d \boldsymbol{x} \\
& =\lim _{t \rightarrow+\infty} \int_{C}\left|\left(e^{-i H_{o c}(t)} S_{c} f\right)(\boldsymbol{x})\right|^{2} d \boldsymbol{x} \\
& =\lim _{t \rightarrow+\infty}\left|\frac{m}{t}\right|^{3} \int_{C}\left|\widetilde{S_{c} f}\left(\frac{m \boldsymbol{x}}{t}\right)\right|^{2} d \boldsymbol{x} \\
& =\int_{C}\left|\widetilde{S_{c} f}(\boldsymbol{k})\right|^{2} d \boldsymbol{k}
\end{aligned}
$$

We now want to show that $P(f, C)$ of (29) can actually be obtained by the prescription (22). In order to do this, we need to analyse the asymptotic behavior as $t \rightarrow+\infty$ of the function

$$
F(t)=e^{-2 i H t} e^{i H_{0} t} f .
$$

When $H$ is given by (24).

We will see that

$$
\lim _{t \rightarrow+\infty}\left\|F(t)-e^{-i H_{o} t} e^{-2 i H_{o c}^{\prime}(t)} S_{c} f\right\|=0 .
$$

This will show (Lemma 3 ) that the ppd determined by $F(t)$ can asymptotically be replaced by the ppd determined by $e^{i H_{o} t} e^{-2 i H_{o c}^{\prime}(t)} S_{c} f$. This latter ppd, however, can asymptotically be replaced by $\left|\frac{m}{t}\right|{ }^{3}\left|\widetilde{S_{c} f}\left(\frac{m \boldsymbol{x}}{t}\right)\right|^{2}$. [This fact is an immediate consequence of $(26)$ if we merely replace there the charge $e$, occurring in the definition of $H_{o c}^{\prime}(t)$ by $2 e$, this replaces $H_{o c}^{\prime}(t)$ by $2 H_{o c}^{\prime}(t)$ and gives us the desired result.] This, of course, implies that the prescription (22) gives the same result as the prescription (29).

We state explicitly a fact that is implicit in the preceding discussion: if the initial wave function is $f$, then at large positive times the ppd for the particle can be replaced by $\left|\left(e^{-2 i H t} e^{i H_{0} t} f\right)(\boldsymbol{x})\right|^{2}$ when integrating over any measurable set: this is because the ppd determined by the wave-function $e^{-i H t} \Omega_{c}^{-} f$ can be replaced by the ppd of $e^{-i H_{o c}(t)} S_{c} f$, 
which can be replaced by $\left|\frac{m}{t}\right|{ }^{3} \mid \widetilde{S_{c} f}\left(\frac{m \boldsymbol{x}}{t}\right)^{2}$, which can be replaced, according to the last paragraph, by $\left|\left(e^{-2 i H t} e^{i H_{o} t} f\right)(\boldsymbol{x})\right|^{2}$.

In order to prove (31), we rewrite this equation as

$$
\lim _{t \rightarrow+\infty} e^{i H_{o} t} F(t)-e^{-2 i H_{o c}^{\prime}(t)} S_{c} f \|=0 \text {. }
$$

Now because of the intertwining relations [5]

we have

$$
e^{i I I t} \Omega_{c}^{ \pm}=\Omega_{c}^{ \pm} e^{i H_{0} t}
$$

$$
e^{i H_{0} t} S_{c}=S_{c} e^{i H_{0} t} .
$$

It follows that any bounded function of $H_{0}$ commutes with $S_{c}$, and in particular, since $H_{o c}^{\prime}(t)$ is a function of $H_{o}$,

$$
e^{-i H_{o c}^{\prime}(t)} S_{c}=S_{c} e^{-i H_{o c}^{\prime}(t)}
$$

Thus, we must show that

$$
\lim _{t \rightarrow+\infty}\left\|e^{i H_{o} t} F(t)-e^{-i H_{o c}^{\prime}(t)} S_{c} e^{-i H_{o c}^{\prime}(t)} f\right\|=0,
$$

or, what is the same thing,

$$
\lim _{t \rightarrow+\infty}\left\|\left(e^{i H_{o c}^{\prime}(t)} e^{i H_{0} t} F(t) e^{i H_{o c}^{\prime}(t)}-S_{c}\right) e^{-i H_{o c}^{\prime}(t)} f\right\|=0 .
$$

Using (27), the fact that $H_{o c}^{\prime}(t)=-H_{o c}^{\prime}(-t)$, and the fact that the ranges of $\Omega_{c}^{ \pm}$are the same, it is not difficult to see that

$$
\lim _{t \rightarrow+\infty} e^{i H_{o c}^{\prime}(t)} e^{i H_{o} t} F(t) e^{i H_{o c}^{\prime}(t)}=S_{c} .
$$

(38) does not, of course, imply (37) although it suggests why (37) is true. In order to prove (37), one has to resort to the same type of detailed estimates as were given in [5] to prove the relations (27). An indication of the proof of (37) is given in Appendix I.

We summarize the preceding results in

Theorem 2. If $H$ is the Coulomb Hamiltonian (24), and $f$ is the initial state of a particle whose motion is governed by this Hamiltonian, then the ppd determined by the wave-function $e^{-i H t} \Omega_{c}^{-} f$ of the particle can for large $t$ be replaced by the ppd determined by $e^{-2 i H t} e^{i H_{o} t} f$, just as in Theorem 1, and the probability for scattering into a cone $C$ is correctly given by (22).

\section{Conclusion}

We have dealt with scattering by:

a) Any potential for which $\Omega^{ \pm}$exist, and $R^{+}=R^{-}$. That is, any potential for which $\Omega^{ \pm}$exist and the $S$-matrix is unitary. [Any potential $V \in \mathscr{L}^{1}\left(\mathbb{R}^{3}\right) \cap \mathscr{L}^{2}\left(\mathbb{R}^{3}\right)$ satisfies these conditions.]

b) Coulomb potentials and constant potentials. 
In both cases a) and b) we have seen that if the initial state of the particle is $f$, then the ppd of the particle can be replaced by

$$
\left|\left(e^{-2 i H t} e^{i H_{o} t} f\right)(\boldsymbol{x})\right|^{2} \quad \text { as } \quad t \rightarrow+\infty .
$$

This in turn allowed us to compute the probability $P(f, C)$ for scattering: into a cone using the single formula

$$
P(f, C)=\lim _{t \rightarrow+\infty} \int_{C}\left|\left(e^{-2 i H t} e^{i H_{o} t} f\right)(\boldsymbol{x})\right|^{2} d \boldsymbol{x} .
$$

Eq. (39) provides a simple expression for dealing simultaneously with cases which previously required different (and, in the case of the Coulomb potential, far more complicated) formulas.

The formulas given here for the asymptotic ppd and the probability for scattering into a cone provide a suggestion for a way to analyse scattering problems in other cases in which the Møller wave-matrices do not exist.

In a forthcoming paper, we will show how (39) can be generalized to problems involving $n$-body multichannel scattering.

\section{Appendix I}

In order to prove the result (37), we write

$$
\begin{aligned}
e^{i H_{o c}^{\prime}(t)} e^{i H_{o} t} F(t) e^{i H_{o c}^{\prime}(t)} & \\
& -S_{c}=\left(e^{i H_{o c}^{\prime}(t)} e^{i H_{o} t} F(t) e^{i H_{o c}^{\prime}(t)}-e^{i H_{o c}^{\prime}(t)} e^{i H_{o} t} e^{-i I t} \Omega_{c}^{-}\right) \\
& +\left(e^{i H_{o c}^{\prime}(t)} e^{i H_{o} t} e^{-i H t} \Omega_{c}^{-}-\Omega_{c}^{+*} \Omega_{c}^{-}\right)
\end{aligned}
$$

and substitute into (37). Then, using the triangle inequality, we can dominate the norm on the left-hand side of (37) by the sum of two terms, one of which is

$$
g(t)=\left\|\left(e^{i H_{o c}^{\prime}(t)} e^{i H_{o} t} e^{-i H^{t}} \Omega_{c}^{-}-\Omega_{c}^{+*} \Omega_{c}^{-}\right) e^{-i H_{o c}^{\prime}(t)} f\right\| .
$$

We shall prove that this term tends to zero as $t \rightarrow \infty$, leaving the proof for the other term to the reader.

We have

$$
\begin{aligned}
g(t) & =\left\|\left(\Omega_{c}^{-}-e^{i H t} e^{-i H_{o} t} e^{-i H_{o c}^{\prime}(t)} \Omega_{c}^{+*} \Omega_{c}^{-}\right) e^{-i H_{o c}^{\prime}(t)}\right\|_{\|} \\
& =\left\|\left(\Omega_{c}^{+}-e^{i H t} e^{-i H_{o} t} e^{-i H_{o c}^{\prime}(t)}\right) \Omega_{c}^{+*} \Omega_{c}^{-} e^{-i H_{o c}^{\prime}(t)} f\right\| \\
& =\left\|\left(\Omega_{c}^{+}-e^{i H t} e^{-i H_{o} t} e^{-i H_{o c}^{\prime}(t)}\right) e^{-i H_{o c}^{\prime}(t)} S_{c} f\right\| .
\end{aligned}
$$

We will now replace $S_{c} f$ by a $C$-function [5] $h$ and prove convergence to zero. The convergence to zero of the right-hand side of (A 3) is an easy consequence of the convergence to zero with $S_{c} f$ replaced by 
a $C$-function $h$, since the $C$-functions are dense in $\mathscr{L}^{2}$, and the operators involved are bounded. We have

$$
\begin{aligned}
& \left\|\left(\Omega_{c}^{+}-e^{i H t} e^{-i H_{o} t} e^{-i H_{o c}^{\prime}(t)}\right) e^{-i H_{o c}^{\prime}(t)} h\right\| \\
& \quad \leqq \int_{t}^{\infty}\left\|\frac{d}{d S}\left(e^{i H S} e^{-i H_{o} S} e^{-i H_{o c}^{\prime}(S)}\right) e^{-i H_{o c}^{\prime}(t)} h\right\| d S=r(t) .
\end{aligned}
$$

Analysing the behavior of the function $e^{\left.-i H_{o c}^{\prime}{ }^{(} t\right)} h$ as in [5], it is possible to obtain the bound

$$
r(t) \leqq C(\log |t|)^{n} \int_{t}^{\infty} \frac{(\log |S|)^{m}}{S^{3 / 2}} d S
$$

for some constant $C$ and integers $m$ and $n$. (A 5) shows that

$$
r(t) \underset{t \rightarrow \infty}{\longrightarrow} 0
$$

as desired.

\section{References}

1. Kato, T.: Trans. Am. Math. Soc. 70, 195 (1951).

2. Schwartz, L.: Theorie des Distributions. Vol. II, p. 89 ff. Paris: Hermann \& Cie 1957.

3. Kuroda, S. T.: Nuovo Cimento 12, 431 (1959).

4. Green, T. A., and O. E. Lanford, III: J. Math. Phys. 1, 139 (1960).

5. Dollard, J.: J. Math. Phys. 5, 729 (1964).

J. D. Dollard

Department of Mathematics

Yale University

Box 2155, Yale Station

New Haven, Connecticut 06520, USA 